\title{
A Novel Algorithm for the Optimal Network Selection Scheme in the User Centric 4G networks
}

\author{
Manu Mohan C M \\ $M$ Tech Scholar \\ FISAT, Angamaly
}

\author{
Benoy Abraham \\ Assistant Professor, ECE Dept. \\ FISAT, Angamaly
}

\begin{abstract}
Since the advancement of current cellular systems and technology is augmented towards $4 \mathrm{G}\left(4^{\text {th }}\right.$ Generation) and beyond, the need for efficiency in terms of the Quality of Service(QoS) and reducing the signaling overhead between the mobile node and the various other equipment providing services within the core network becomes critical. Selection of application as per the user preference has much importance in the $4 \mathrm{G}$ networks. In $4 \mathrm{G}$ networks it is necessary to integrate WLAN (IEEE 802.11) systems with currently existing UMTS, GPRS systems which provide for wider mobility requirements, if the user requires obtaining a substantial amount of bandwidth within a hot-spot province. In this paper, a selection algorithm has been proposed which provides a better way to implement user preferences as per the features of $4 \mathrm{G}$. The proposed algorithm uses a distance function to generate an ordered list of various access technologies called networks in a particular region according to multiple user preferences and level of interest. Further facilitating an optimal choice of AP (Access Point) for the MN (Mobile Node) to link up with, whenever it moves from one APs coverage area to another.
\end{abstract}

\section{General Terms}

Distance Function, Access Point Selection.

\section{Keywords}

4G Networks., QoS, , Mobile Node.

\section{INTRODUCTION}

It is expected that the $4^{\text {th }}$ generation $(4 \mathrm{G})$ networks will ensure seamless interoperability and interconnection with other mobile devices and technologies in heterogeneous networks since the next generation systems will support multimode, multi- access and reconfigurable devices.
From the service point of view, it foresees that $4 \mathrm{G}$ will be mainly focused on personalized services [5] with ultra-high speed wireless broadband system [4]. Therefore, it emphasizes the heterogeneity of networks and new service infrastructures, rather than increased bandwidth. Hence they are expected to provide all IP-based services for heterogeneous wireless access technologies [6], assisted by mobile IP. Two major challenges in developing such heterogeneous network infrastructure are QoS provisioning and security services for mobile users' communication flows.

A universal consensus on what is going to be $4 \mathrm{G}$ is not yet reached in the industry or the literature; there is a reasonable understanding of some characteristics of $4 \mathrm{G}$ mobile networks [6].

Some of the accepted characteristics are:

- $\quad$ All-IP based network architecture;

- Higher bandwidth;

- Support for different access networks, including WLAN technologies like IEEE 802.11;

- Full integration of "hot spot" and "cellular";

- Support for multimedia applications.

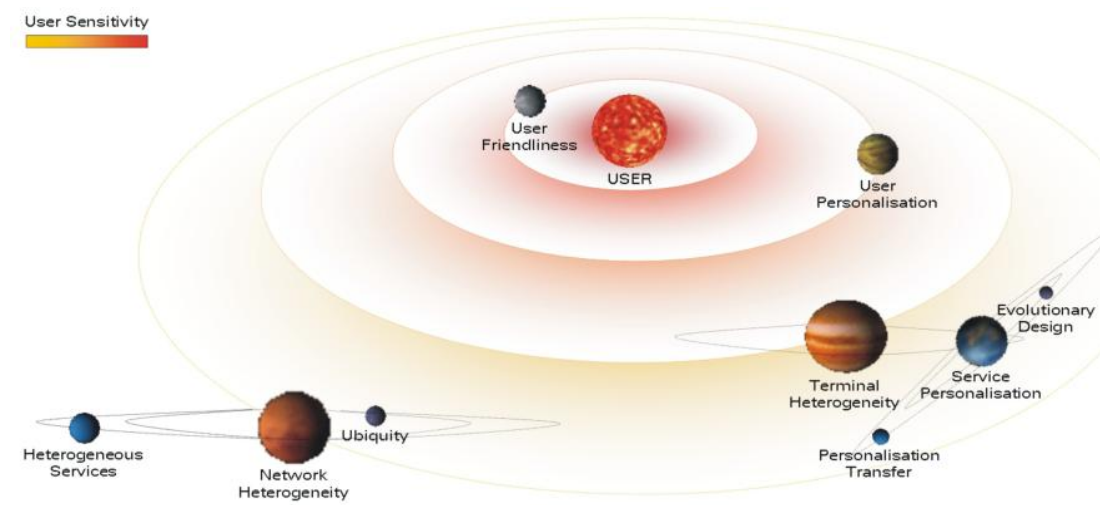

Fig. 1: User Centric System 
The $4 \mathrm{G}$ can provide all available services to even highly mobile people by total convergence of the wireless mobile and wireless access communication in a ubiquitous manner. It is not just IP end-to-end communication but over-the-air packet switching. Faster hand-off is accomplished due to high speed transmission. 4G communication ranges at $100 \mathrm{Mbps}$ while moving and $1 \mathrm{Gbps}$ while stationary. The major advantage of Next Generation Networks (NGN) is the interoperability of different standards hampering global mobility and service portability. This interconnected network has lead to the evolution of a new paradigm for future communications, namely "Always Best Connected" (ABC) [3], in comparison to typical $2 \mathrm{G}$ and $3 \mathrm{G}$ concept of "Always Connected".

\section{EXISTING SYSTEM}

Since a final conformity on what features characterize $4 \mathrm{G}$ mobile system is yet to be reached; this topic becomes a hot research area presently. Several methods have been proposed as a solution to the network selection problem in $4 \mathrm{G}$. Quality of Service (QoS) based network selection technique [3] is the one among them. According to this, all the QoS parameters of the network are listed before selecting them, which includes bandwidth, security, connectivity, cost of service etc. A distance function based algorithm is performed on the ordered list of networks which shows the rank of the networks which provide the best of particular parameters. Weights are assigned to these ranks as per user's priority at the moment. The best network is selected from these values. The only shortcoming would be a bit of overhead with all the calculations.

In the paper [8] an efficient load balancing based AP selection algorithm which considers the direction of advancement of the Mobile Node is described, and hence is able to extract the optimal node for the User Equipment to link up with as it moves.

\section{PROPOSED SYSTEM}

In $4 \mathrm{G}$ systems, each user has his/her own requirements that are unique. User also demands that the network selection should be as per his/her requests, even during the handoff process. The user need not specify his/her specific requirements at the time of handoff. The device must also be able to select the network that satisfies the current session's QoS requirements. Thus, a novel network discovery and selection mechanism must be provided.

Network selection signifies to explore the available networks in a region and choose the best among them. Since the user has more preference on the selection of service depending on the application, among the available networks according to his/her requirements a novel network selection mechanism is being proposed such that the selected network satisfies the current session's QoS requirements. Each network would be assigned a weight which is based on the QoS parameters and factors that it provides and satisfies the end user requirements.
Then it's required to compute the sum of all weight assigned to a particular network which is then normalized within the range of 0-10. A network scoring maximum shall be the best available network while a network scoring less than a specified threshold shall be ignored and similarly, a network gaining a zero weight shall be straightaway discarded.

\subsection{Cost of Services}

The applications based on the user preferences are under different billing schemes. Customized network selection scheme demands higher costs for higher QOS requirements. It is continuously updated by the user according to his/her profile.

\subsection{Bandwidth Utilization}

The user chooses a service provider or network based on the amount of data transfer to utilize the bandwidth efficiently. This has to be stored in history because a hand-off is necessary when the bandwidth degrades beyond certain limit. This value is noted as Bandwidth Factor. It is also proportional to the total duration of the session.

\subsection{Security}

Security level offered and areas covered are another important attributes. A user interested in accessing a network to perform e-transaction would be more concerned about the level of security provided by the available networks. However, other user, moving frequently might give preference to the network which offers uniform signal strength and/or results in less number of handovers.

\subsection{Call Drop during Handoff}

During Handoff, Call Drop Probability of the user may change according to the type of application. It affects the selection of hard and soft handoff. It may also affect the selection of networks in terms of priority of handoff calls.

\subsection{Minor Network parameters}

Network selection may also depend on certain parameters such as battery level. It may be well considered and it varies depending on the type of application.

\section{PROPOSED ALGORITHM}

Let us consider the set of available networks to the choices of the user are represented as, $\mathrm{N}=\{\operatorname{Net}(1)$, Net (2), Net (3), Net (4), Net (5) $\} \ldots$ The user-specified parameters are represented as $\mathrm{P}=\{\mathrm{P}(1), \mathrm{P}(2), \mathrm{P}(3), \mathrm{P}(4)\} \ldots$ The user may be more customized by selecting (say) one or more essential entities like $\mathrm{P}=\{$ Bandwidth, Security level $\}$. The analysis of the available access technologies is also made. For Net1, P1= $\{$ free, low $\}$, for Net2, P2 $=\{$ medium, high $\}$ and for Net3, P3= $\{$ high, medium $\}$ and the ordered list is done as $\mathrm{XBW}=\{$ Net1, Net2, Net 3$\}$ and XSECURITY $=\{$ Net1, Net 3, Net 2$\}$ and then ranked according to different parameters. 
The weighted distance function is customized with the user interest level parameter. It is because an adverse condition management application is more concerned about the Bandwidth for the faster data rate rather than cost or security level. In this multi-attribute distance function, the parameters are normalized to values ranging from zero to one A network with billing parameter of 0.9 is better compared to that of 0.5 values.

Table 1: Network parameters and their features

\begin{tabular}{|l|l|l|l|}
\hline & USER 1 & USER2 & USER3 \\
\hline APPLICATION & $\begin{array}{l}\text { LIVE } \\
\text { VIDEO }\end{array}$ & $\begin{array}{l}\text { e- } \\
\text { TRANSACTION }\end{array}$ & CALLING \\
\hline Cost & Medium & High & Low \\
\hline Bandwidth & High & Medium & Medium \\
\hline Security & Low & High & Low \\
\hline Call Drop & Medium & Low & Medium \\
\hline
\end{tabular}

Table 2: User Specified Parameters

\begin{tabular}{|l|l|l|l|}
\hline & Net1 & Net2 & Net3 \\
\hline $\begin{array}{l}\text { Cost of } \\
\text { services }\end{array}$ & Medium & Low & High \\
\hline Bandwidth & High & Medium & Low \\
\hline Security & Medium & High & Low \\
\hline $\begin{array}{l}\text { Call Drop } \\
\text { Prob. }\end{array}$ & Low & Medium & Medium \\
\hline
\end{tabular}

\section{NETWORK SELECTION ALGORITHM}

Input: The parametric list of available networks

Output: Optimally ordered list of networks

Step 1: Assign position score for each network in each of the $\mathrm{n}$ lists.

Step 2: Find the sum of position scores to obtain a resultant value.

Step 3: Add necessary weights as per the user demand Step 4: Sort the obtained list
Step 5: for $\mathrm{i}=1$ to $\mathrm{m}$

for $\mathrm{j}=1$ to $n$

$S(j)=$ the no. of networks whose score of parameter $x$ is ranked below $\mathrm{j}$ in sorted list

Step 6: Sort $\mathrm{N}$ (j) in decreasing order where $\mathrm{j}=1$ to $\mathrm{m}$ Stop

Fig. 2: Flowchart of proposed network selection scheme

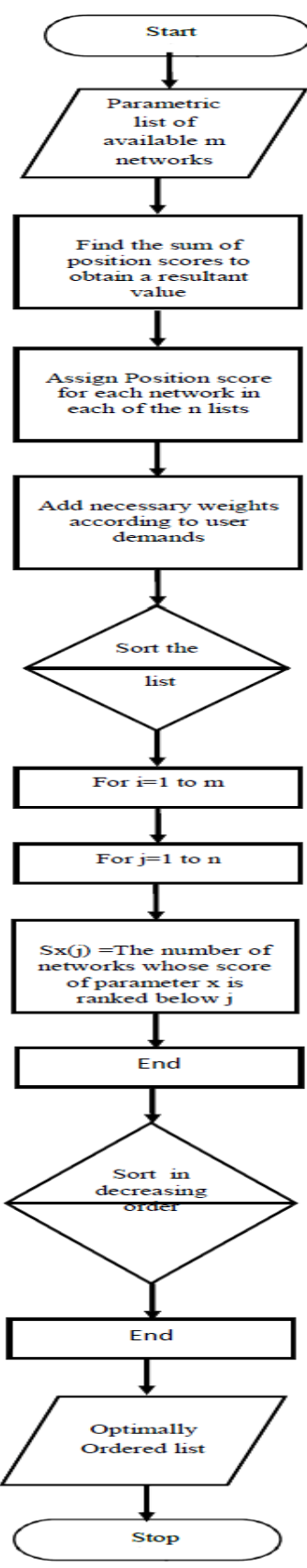

\section{TESTING AND RESULT OF THE PROPOSED ALGORITHM}

In order to employ the algorithm, a test area is created. In this test environment, 5 different networks (Net1, Net2, Net3, Net4 and Net5) have been assumed to coexist within 3 regions R1, R2 and R3. Three different users carrying $4 \mathrm{G}$ devices and 
are required to perform different services. Table 1 gives the parameters and features of the test networks and table 2 contains various user preferences along with the level of interest in multiple parameters as per their requirements and needs. As the entire test area has been divided into three regions: R1, R2, and R3. Net1 and Net5 are available in all the regions. Net2 is available in regions except R1. Net3 is available in regions except R2. Net4 is available in regions except R3. User1 and user2 are in R1 initially and user1 is moving towards $\mathrm{R} 2$ and user2 towards $\mathrm{R} 2$ and then to $\mathrm{R} 3$. User3 is in R3 initially and moving to R1.

The optimum network is selected using proposed algorithm presented in section IV. As the user moves from one region to another, the networks available in the new region are again searched for optimal solution as per the user needs and preferences and, if required, the user is connected to a most suitable network. Three network selection schemes have been compared namely, cost based, bandwidth based and security based. When user preference is towards the cost function the network with low cost is selected and when the user moves from one region to other it performs the selection algorithm again and selects the optimum among the available networks. If the current network is available in the subsequent region no switching between networks is occurs

\section{CONCLUSION}

The paper proposes a novel algorithm for optimal network selection based on multiple user preferences under heterogeneous network. In future $4 \mathrm{G}$ mobile environments, various access technologies will coexist, complementing each other. Therefore, a network selection mechanism is required to help mobile users choose the best network; that is, one that provides always best connected $(\mathrm{ABC})$ that suits users needs, and changes, if conditions changes. Thus a novel network selection mechanism which selects the best network based on QoS parameters is proposed.

The proposed algorithm uses a distance function to generate an ordered list of various available access networks in a particular region according to the multiple user preferences and level of interest. In case of handoff due to the movement of the user from one region to another region, the process is repeated to find again an appropriate network from all the available networks in the new region. The results clearly show that the proposed algorithm always best connect the user, as per his preferences of QoS parameters in a 4G System.

\section{REFERENCES}

[1] L. Suciu, J.M. Bonnin, A survey of multi-criteria network selection algorithms, Global Mobile Congress, 2004 Shanghais.

[2] Bader, F.; Pinart, C.; Christophi, C.; Tsiakkouri, E.; Ganchev, I.;Friderikos, V.; Bohoris, C.; Correia, L.; Ferreira, L., User-centric analysis of perceived QoS in 4G IP mobile/wireless networks Personal, Indoor and Mobile Radio Communications", 2003. PIMRC 2003. $14^{\text {th }}$ IEEE Proceedings on Volume 3, Issue , 7-10 Sept. 2003 Page(s): 2047 - 2053 vol.3 Digital Object Identifier 10.1109/PIMRC.2003.1259074

[3] DJens Zander, "Key Issues in 4th Generation Wireless Systems \& Infrastructures": Royal Institute of Technology, Stockholm, Sweden, Consultation Workshop, European Commission, Brussels, May 2000.

[4] E. Bohlin, S. Lindmark, J. Bjrkdahl, A. Weber, B. Wingert, P. Ballon, "The Future of Mobile Communications in the EU: assessing the Potential of 4G”, ESTO Publications, February, 2004.

[5] J. M. Pereira, "Fourth Generation: Now, it is Personal", Proceedings of the 11th IEEE international Symposium on Personal, Indoor and Mobile Radio Communications (PIMRC), London, UK, September 2000

[6] Xiaoming Fu, Dieter Hogrefe, Sathya Narayanan, Rene Soltwisch, "QoS and Security in 4G Networks" First annual global mobile congress, shanghai, China, October, 2004.

[7] Frattasi, S. Aalborg Univ. Fathi, H.; Fitzek, F.H.P.; Prasad,; Katz, M.D. "Defining 4G technology from the users perspective" IEEE network, Jan.-Feb. 2006

[8] I. S. Misra, A. Banerjee, READER, ETCE Deptt., Jadavpur University, Kolkata - 700032, India, A Novel Load Sensitive Algorithm for AP selection in 4G Networks. 